\begin{tabular}{|c|l|}
\hline Title & Conductance of single 1,4 disubstituted benzene molecules anchored to Pt electrodes \\
\hline Author(s) & Kiguchi, Manabu; Miura, Shinichi; Hara, Kenji; Sawamura, Masay a; Murakoshi, Kei \\
\hline Citation & $\begin{array}{l}\text { A pplied Physics Letters, 91/5), 053110 } \\
\text { https://doi.org/10.1063/2757592 }\end{array}$ \\
\hline Issue Date & 2007-07-30 \\
\hline Doc URL & http://hdl.handle.net/2115/29648 \\
\hline Rights & Copyright $\odot 2007$ American Institute of Physics \\
\hline Type & article \\
\hline File Information & APL91-05.pdf \\
\hline
\end{tabular}

Instructions for use 


\title{
Conductance of single 1,4-disubstituted benzene molecules anchored to Pt electrodes
}

\author{
Manabu Kiguchi ${ }^{\text {a) }}$ \\ Division of Chemistry, Graduate School of Science, Hokkaido University, Sapporo 060-0810, Japan \\ and PRESTO, Japan Science and Technology Agency, Sapporo 060-0810, Japan \\ Shinichi Miura, Kenji Hara, Masaya Sawamura, and Kei Murakoshi \\ Division of Chemistry, Graduate School of Science, Hokkaido University, Sapporo 060-0810, Japan
}

(Received 15 April 2007; accepted 20 June 2007; published online 30 July 2007)

\begin{abstract}
The authors have studied the conductance of a 1,4-disubstituted isocyanide(-NC) or thiol(-SH) benzene molecule anchored to two Pt electrodes. A single molecular junction showing a well-defined conductance value $\left(\sim 3 \times 10^{-2} G_{0}, G_{0}=2 e^{2} / h\right)$ was fabricated with the Pt electrodes. The conductance of the molecular junction was one order higher than the previously documented value using Au electrodes. These observations could be explained by differences in the local density of states of the contact metal atom at the Fermi level and the extent of the hybridization and energy difference between the molecular and metal orbitals. Further insight into the binding strengths of the metal-anchoring group bond was obtained by statistically analyzing the stretching length of the molecular junction. (C) 2007 American Institute of Physics. [DOI: 10.1063/1.2757592]
\end{abstract}

There is a growing interest in the electron transport properties through single molecules due to their potential use in ultrasmall electronic devices. ${ }^{1}$ In studying the electron transport properties through a single molecule, it is important to design an appropriate molecule-electrode contact, since the contact plays a decisive role on the electron transport process through the molecule. Conductance measurements have been investigated for various single molecules using scanning tunneling microscope (STM) and mechanically controllable break junctions. ${ }^{1-3}$ In most of the studies, the $\mathrm{Au}-\mathrm{S}$ bond is used to connect molecules to metal electrodes because stable molecular junctions can be easily obtained with this Au-S covalent bond. On the other hand, the conductance of a single molecular junction depends on the extent of the hybridization and energy difference between the molecular and metal orbitals and the local density of states (LDOS) $\rho$ of the contact metal atoms at the Fermi level $E_{F}{ }^{4}$ An effective hybridization and a small energy difference between the molecular and metal orbitals, and also large $\rho$ of the contact metal atom at $E_{F}$ are essential for the molecular junction to achieve high conductivity. Generally, the energy of the molecular orbital varies, depending on the molecule and its anchoring group. The $\rho$ of $\mathrm{Au}$ is relatively low compared with other $d$-orbital metals at $E_{F}$ because of their $s p$ state characteristics. Therefore, the $\mathrm{Au}-\mathrm{S}$ bond is not always the best anchoring group for the single molecular junction showing high conductivity. It is important to develop a pair of metal and anchoring group other than $\mathrm{Au}-\mathrm{S}$ to establish highly conductive single molecular junctions.

In developing anchoring groups, the conductance of single molecules anchored by isocyanide $(-\mathrm{NC}),{ }^{5}$ carboxylic acid $(-\mathrm{COOH}),{ }^{6}$ or amine $\left(-\mathrm{NH}_{2}\right)$ (Ref. 7) anchoring groups to Au electrodes has been studied. Still, the metal of the electrode is restricted to Au. In spite of the variation in the choice of molecules, there have been few studies of single molecular junctions using metals other than Au. Among various metals, we pay attention to Pt for the following three

\footnotetext{
${ }^{a)}$ Electronic mail: kiguti@sci.hokudai.ac.jp
}

reasons. First, $\mathrm{Pt}$ is chemically stable, which is essential for preparing a clean surface on the metal electrodes. Second, air stable thiol and isocyanide self-assembled monolayer (SAM) films are formed on Pt surfaces. ${ }^{8}$ The formation of stable SAMs indicates that the stable $\mathrm{Pt}-\mathrm{S}$ or $\mathrm{Pt}-\mathrm{CN}$ bond is formed and molecules are not decomposed on a Pt surface. Third, the $\rho$ is high for Pt compared with Au at $E_{F}$, since a narrow $5 d$ band is located at $E_{F}$ for $\mathrm{Pt}^{9}{ }^{9}$ The conductance of a single molecule anchored to the Pt electrodes would be higher than that of the Au electrodes due to the high $\rho$ of Pt. Recent theoretical calculations show promise for high conductivity of the molecular junctions with Pt electrodes. ${ }^{10,11}$ The calculated conductance of the molecular junction binding a benzene molecule to Pt electrodes by thiol or isocyanide coordination was estimated to be one order higher than that of the Au electrodes. ${ }^{10,11}$

In the present study, the effect of the metal and anchoring group on the conductance of single molecular junctions was investigated for 1,4-disubstituted (-NC- or $-\mathrm{S}$ ) benzene molecules anchored to two Pt or Au electrodes. The metalanchoring group bond strength was qualitatively estimated by the distance over which the molecular junction was stretched before breakdown. The conductance of the single molecular junction is discussed, considering a simple tunneling model.

The experimental design used in this study was the same as described in our previous report. ${ }^{5}$ A sharp tip was used, comprising a Pt wire (diameter of $\sim 0.25 \mathrm{~mm}$, $>99.9 \%)$. The substrate was $\operatorname{Pt}(111)$, prepared by flame annealing and quenching method. ${ }^{12}$ The measurements were carried out in tetraethyleneglycol dimethyl ether (tetraglyme). The solution contained $1 \mathrm{~m} M$ 1,4-diisocyanobenzene and 1,4-benzenedithiol. A STM tip was repeatedly moved into and out of contact with a metal substrate at a rate of $50 \mathrm{~nm} / \mathrm{s}$ in the solution. Respective molecules terminated with two isocyanide or thiol anchoring groups can bridge the tip and the substrate electrodes via their anchoring groups. Conductance was measured during the breaking process under an applied bias of $20 \mathrm{mV}$ between the tip and substrate. 

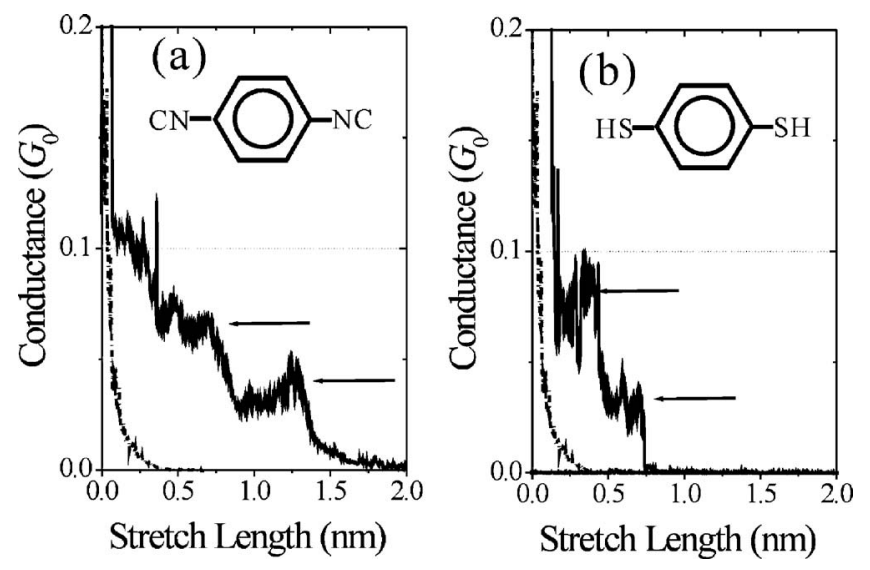

FIG. 1. Typical conductance traces of Pt contacts during breaking the contact in a tetraglyme solution containing (a) 1,4-diisocyanobenzene and (b) 1,4-benzenedithiol. The dotted line shows the result in the absence of molecules.

All statistical data were obtained from a large number (over 1000) of individual conductance traces.

Figures 1 and 2 show the typical conductance traces and histograms of the Pt contacts during breaking the contact in a tetraglyme solution, with and without $1 \mathrm{~m} M 1,4-$ benzenedithiol or 1,4-diisocyanobenzene. In the absence of molecules, neither plateaus nor peaks were observed both in the conductance traces and histograms (see Figs. 1 and 2). In solution containing the molecules, there are two characteristics in the conductance traces. First, the conductance trace shows a conductance fluctuation, which would originate from the structural transformation of the molecular junction. ${ }^{13}$ Second, the trace shows a plateau in which the conductance is nearly constant (arrow in Fig. 1), and the conductance value of the plateau is an integer multiple of $\sim 3 \times 10^{-2} G_{0}\left(=2 e^{2} / h\right)$. The corresponding histogram shows a feature at $3 \times 10^{-2} G_{0}$ [see Figs. 2(a) and 2(b)]. The last plateau before the contact is broken in the conductance trace and a peak observed in the conductance histogram likely originates from the formation of stable single Pt/1,4benzenedithiol/Pt and Pt/1,4-diisocyanobenzene/Pt junctions. The conductance of a single molecular junction was determined to be $3 \times 10^{-2}\left( \pm 1 \times 10^{-2}\right) G_{0}$ for both molecular junctions. A benzene molecule is possibly bound to $\mathrm{Pt}$ atoms via
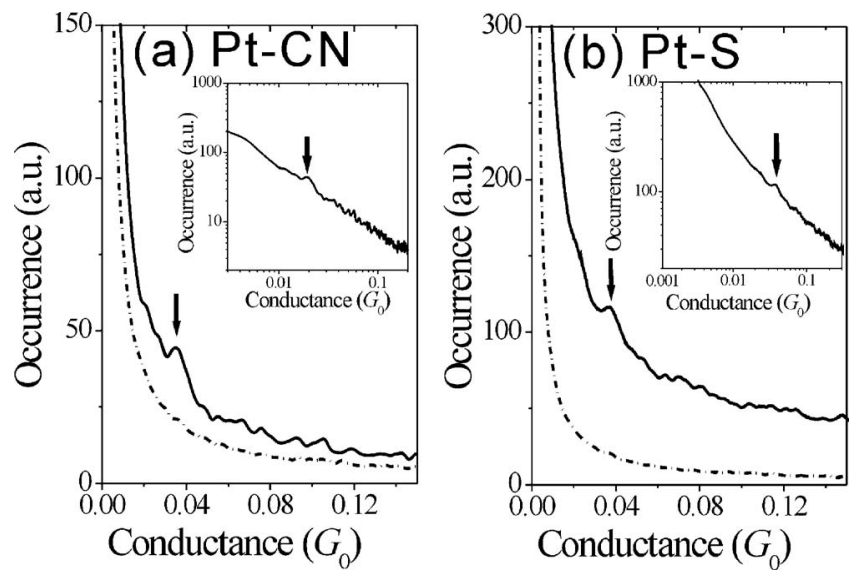

FIG. 2. Conductance histogram of Pt point contacts broken in the tetraglyme solution containing (a) 1,4-diisocyanobenzene and (b) 1,4-benzenedithiol. The dotted line shows the result in the absence of molecules. The insets

show the conductance histograms shown on a log-log plot. to compare the conductance of single molecular junction of
Downloaded 15 Oct 2007 to 133.87 .26 .100 . Redistribution subject to AlP license or copyright, see http://apl.aip.org/apl/copyright.jsp

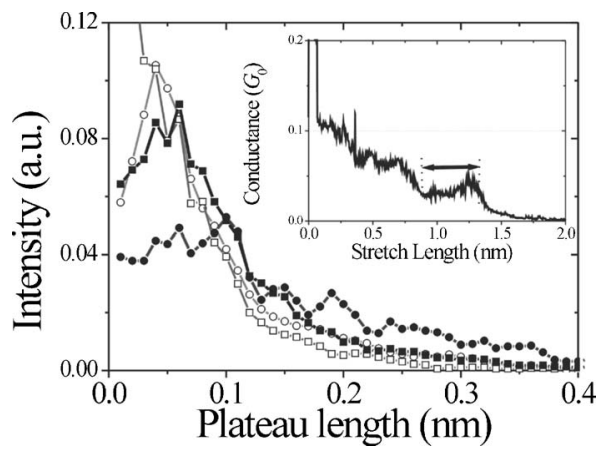

FIG. 3. Distribution of lengths for the last conductance plateau for $\mathrm{Pt}-\mathrm{S}$ (filled circle), $\mathrm{Au}-\mathrm{S}$ (circle), $\mathrm{Au}-\mathrm{CN}$ (box), and Pt-CN (filled box). The inset shows the typical conductance trace for the 1,4-diisocyanobenzene junction on Pt electrodes. The plateau regime is shown by the arrows. The length of the last conductance plateau was defined as the distance between the points (set points) at which the conductance dropped below $4 \times 10^{-2} G_{0}$ and $3.0 \times 10^{-2} G_{0}$ for the molecular junctions with the $\mathrm{Pt}-\mathrm{S}$ bonds. The set points were $4.3 \times 10^{-2} G_{0}$ and $2.7 \times 10^{-2} G_{0}$ for the $\mathrm{Pt}-\mathrm{CN}$ bond, $4.8 \times 10^{-3} G_{0}$ and $3.0 \times 10^{-3} G_{0}$ for the Au-S bond, and $3.2 \times 10^{-3} G_{0}$ and $2.0 \times 10^{-3} G_{0}$ for the $\mathrm{Au}-\mathrm{CN}$ bond, respectively.

$\mathrm{S}$ atoms for the $\mathrm{Pt} / 1,4-\mathrm{benzenedithiol/Pt} \mathrm{junction.} \mathrm{For} \mathrm{the}$ $\mathrm{Pt} /$,4-diisocyanobenzene/Pt junction, isocyanide coordination via $\mathrm{C}$ atoms may contribute to the junction formation. In the following, these molecular junctions are referred to as the molecular junction with the $\mathrm{Pt}-\mathrm{CN}$ bond and the molecular junction with the $\mathrm{Pt}-\mathrm{S}$ bond, respectively. In our previous study, the conductance of the molecular junction was determined to be $4 \times 10^{-3}\left( \pm 1 \times 10^{-3}\right) G_{0}$ for the junction with the $\mathrm{Au}-\mathrm{S}$ bond and $3 \times 10^{-3}\left( \pm 1 \times 10^{-3}\right) G_{0}$ for the junction with the $\mathrm{Au}-\mathrm{CN}$ bond. Present results prove that the conductance of the single molecule junction with the Pt electrodes is one order higher than that with the Au electrode.

Before discussing the conductance of the single molecular junction in detail, the metal-anchoring group bond strength is discussed by statistical analysis of the conductance trace. Since the last plateau corresponds to a single molecular junction, ${ }^{5,14}$ the length of the last plateau should be the distance over which the single molecular junction can be stretched before breakdown (breakdown distance). The breakdown distance reflects the stability of the molecular junction and the strength of the metal-anchoring group bond. ${ }^{5}$ To evaluate characteristics of the breakdown distance quantitatively, the distribution of the breakdown distance of the molecular junction was investigated (Fig. 3). For comparison, the results obtained by the $\mathrm{Au}-\mathrm{S}$ and $\mathrm{Au}-\mathrm{CN}$ bonds in our previous reports ${ }^{5}$ are also shown in the figure. The average length of the molecular junction was 0.16 $( \pm 0.02) \mathrm{nm}$ for the junction with the $\mathrm{Pt}-\mathrm{S}$ bond, 0.11 $( \pm 0.01) \mathrm{nm}$ for the Pt-CN bond, $0.090( \pm 0.009)$ for the $\mathrm{Au}-\mathrm{S}$ bond, and $0.064( \pm 0.006) \mathrm{nm}$ for the $\mathrm{Au}-\mathrm{CN}$ bond. Since the stretching length reflects the bond strength of the molecular junctions, ${ }^{5}$ the present results suggest that the strength of the metal-anchoring group bond decreased in the order of $\mathrm{Pt}-\mathrm{S}, \mathrm{Pt}-\mathrm{CN}, \mathrm{Au}-\mathrm{S}$, and $\mathrm{Au}-\mathrm{CN}$.

Based on the above experimental results, the conductance of the single molecular junction will now be discussed. The experimentally obtained conductance values of a single molecular junction can differ by two orders of magnitude among various reports, even for the same molecule, due to different experimental conditions. ${ }^{2,15}$ Generally, it is difficult to compare the conductance of single molecular junction of to AIP license or copyright, see http://apl.aip.org/apl/copyright.jsp 
different molecules measured under different conditions. On the other hand, statistical analysis of the present data showed that the conductance values of a single molecular junction measured under the same conditions could be determined to a precision of $30 \% .^{5}$ Thus, the comparison of the conductance values of the single molecular junction with $\mathrm{Au}$ and $\mathrm{Pt}$ using our results measured under the same condition may provide information on the metal dependence. Our series of recent studies ${ }^{5}$ show that the conductance of the molecules anchored to the Pt electrodes was one order higher than those of the Au electrodes, while the conductance showed little dependence on the choice of the anchoring groups. Recently, the conductance of single benzene molecule anchored by $\mathrm{S}$ or NC anchoring groups to $\mathrm{Au}$ and Pt electrodes has been calculated, considering the tunneling model ${ }^{10,11}$ Similar tendencies in the conductance differences among the single molecular junctions with the $\mathrm{Au}-\mathrm{S}, \mathrm{Au}-\mathrm{CN}, \mathrm{Pt}-\mathrm{S}$, and $\mathrm{Pt}-\mathrm{CN}$ bonds were observed for both calculations and in our experimental results, although the calculated conductance values are about ten times higher than the experimental ones. Observed conductance differences in the systems of the 1,4disubstituted $(-\mathrm{CN}$ or $-\mathrm{SH})$ benzene molecules anchored to two Pt or Au electrodes can be discussed, considering the tunneling model.

In a simple tunneling model, ${ }^{4}$ the conductance $[G(E)]$ of the single molecular junction is given by $T(E)$ $=A\left(\left(\beta^{2} \rho / E-\varepsilon_{i}\right)\right)^{2}$, where $A, \beta, \rho$, and $\varepsilon_{\mathrm{i}}$ are the constant, hopping integral between the molecule and metal orbitals, local density of states value of the contact metal atom at the $E_{F}$, and the energy of the $i$ th molecular orbital, respectively. Here, $\beta$ increases with the bond strength. ${ }^{5}$ Large $\rho, \beta$, and small $E_{F}-\varepsilon_{i}$ are essential for molecular junctions showing high conductivity. The $\rho$ of Pt is much larger than the $\rho$ of Au at the $E_{F}{ }^{8,16}$ Statistical analysis of the stretching length of the molecular junction showed that the binding energy of $\mathrm{Pt}-\mathrm{S}$ and $\mathrm{Pt}-\mathrm{CN}$ bonds was larger than that of $\mathrm{Au}-\mathrm{S}$ and $\mathrm{Au}-\mathrm{CN}$ bonds, suggesting that the $\beta$ of the junction with the Pt electrode is larger than that with the Au electrode. Due to relatively large $\rho$ and $\beta$ for the junction with the Pt electrode, the conductance of the molecular junction with the Pt electrode would be one order higher than that with the Au electrode. Higher conductivity in the Pt electrode than that of the $\mathrm{Au}$ observed in the present experiment can be explained by these contributions of $\rho$ and $\beta$.

Here, we briefly comment on the fact that the conductance of the molecular junction with the Pt-S bond was close to that with the $\mathrm{Pt}-\mathrm{CN}$ bond. The value of $E_{F}-\varepsilon_{i}$ varies with the choice of the anchoring group, and it would also affect the difference in the conductance between the molecular junction with the Pt electrode and Au electrode. The stretching length of the molecular junction showed that the binding energy $(\beta)$ of the $\mathrm{Pt}-\mathrm{S}$ bond was larger than that of the $\mathrm{Pt}-\mathrm{CN}$ bond. Since the conductance is obtained as the product of $\beta^{2}$ and $1 /\left(E_{F}-\varepsilon_{i}\right)$, the close agreement between the conductance of the molecular junction with the Pt-S and $\mathrm{Pt}-\mathrm{CN}$ bonds indicates that energy difference between molecular and metal orbitals would be larger for the junction with the $\mathrm{Pt}-\mathrm{S}$ bond than for the $\mathrm{Pt}-\mathrm{CN}$ bond.
The present experimental study clearly showed that $\rho, \beta$, and $E_{F}-\varepsilon_{i}$ play the decisive role in determining the conductance of the single molecule. In developing an electronic device with a single molecule, it is becoming important to study electron transport through a single molecule with a high degree of functionality. ${ }^{1}$ The three factors depend on the choice of the molecules and the electrode metals. By considering these three factors in choosing the best electrode metal for the individual molecules, the conductance of the single molecule junction would be drastically improved, which leads to a higher performance for the molecular devices. Finally, the effect of the catalytic behavior of Pt on the single molecular junction is briefly discussed. Various catalytic reactions occur on the Pt surface due to its high catalytic reactivity. ${ }^{17}$ If an organic molecule is adsorbed strongly in uncontrolled manner on the Pt electrode, the molecule cannot be used for the single molecular junction. On the other hand, by introducing the anchoring groups to the target molecule, the molecule is bound to the metal surface via the anchoring group. The molecule is in contact with the metal surface having well-defined adsorbed structure. Choosing appropriate anchoring group should be very important to control the adsorption of the molecule for the single molecular junctions.

In conclusion, we fabricated well-defined molecular junctions with $\mathrm{Pt}-\mathrm{S}$ and $\mathrm{Pt}-\mathrm{CN}$ bonds. The conductance of the molecular junctions with the Pt electrodes was one order higher than that with the Au electrodes. A Pt metal is a promising choice for a single molecular junction.

This work was partially supported by a Grant-in-Aid for Scientific Research A (No. 16205026) and Grant-in-Aid for Scientific Research on Priority Areas (No. 17069001) from MEXT and the Asahi Glass Foundation.

${ }^{1}$ N. J. Tao, Nat. Nanotechnol. 1, 173 (2006).

${ }^{2}$ M. A. Reed, C. Zhou, C. J. Muller, T. P. Burgin, and J. M. Tour, Science 278, 252 (1997)

${ }^{3}$ B. Xu and N. J. Tao, Science 301, 1221 (2003).

${ }^{4}$ T. Tada and K. Yoshizawa, ChemPhysChem 12, 1035 (2002).

${ }^{5}$ M. Kiguchi, S. Miura, K. Hara, M. Sawamura, and K. Murakoshi, Appl. Phys. Lett. 89, 213104 (2006).

${ }^{6}$ F. Chen, X. Li, J. Hihath, Z. Huang, and N. Tao, J. Am. Chem. Soc. 128, 15874 (2006).

${ }^{7}$ L. Venkataraman, J. E. Klare, I. W. Tam, C. Nuckolls, M. S. Hybertsen, and M. L. Steigerwald, Nano Lett. 6, 458 (2006).

${ }^{8}$ Z. Li, S. Chang, and R. S. Williams, Langmuir 19, 6744 (2003).

${ }^{9}$ B. Hammer and J. K. Norshov, Nature (London) 376, 238 (1995).

${ }^{10}$ J. M. Seminario, C. E. De La Cruz, and P. A. Derosa, J. Am. Chem. Soc. 123, 5616 (2001).

${ }^{11}$ J. W. Lawson and C. W. Bauschlicher, Phys. Rev. B 74, 125401 (2006).

${ }^{12}$ J. Clavilier, R. Faure, G. Guinet, and R. Durand, J. Electroanal. Chem. Interfacial Electrochem. 107, 205 (1980).

${ }^{13}$ W. Thijssen, D. Djukic, A. Otte, R. H. Bremmer, and J. M. van Ruitenbeek, Phys. Rev. Lett. 97, 226806 (2006).

${ }^{14}$ M. Kiguchi, T. Konishi, and K. Murakoshi, Phys. Rev. B 73, 125406 (2006).

${ }^{15}$ J. He, O. Sankey, M. Lee, N. Tao, X. Lic, and S. Lindsay, Faraday Discuss. 131, 145 (2006).

${ }^{16}$ J. C. Cuevas, J. Heurich, F. Pauly, W. Wenzel, and G. Schon, Nanotechnology 14, R29 (2003).

${ }^{17}$ Handbook of Heterogeneous Catalysis, edited by G. Ertl, H. Knozinger, and J. Weitkamp (Wiley-VCH, Weinheim, 1997). 